\title{
PI3K integrates the action of insulin and leptin on hypothalamic neurons
}

\author{
Allison Wanting Xu, ${ }^{1}$ Christopher B. Kaelin, ${ }^{1}$ Kiyoshi Takeda, ${ }^{2}$ Shizuo Akira, ${ }^{2}$ \\ Michael W. Schwartz, ${ }^{3}$ and Gregory S. Barsh ${ }^{1}$
}

\begin{abstract}
${ }^{1}$ Departments of Genetics and Pediatrics, Stanford University School of Medicine, Stanford, California, USA. ${ }^{2}$ Research Institute for Microbial Diseases, Osaka University, Osaka, Japan. ${ }^{3}$ Department of Medicine, Harborview Medical Center, University of Washington, Seattle, Washington, USA.
\end{abstract}

\begin{abstract}
Central control of energy balance depends on the ability of proopiomelanocortin (POMC) or agouti-related protein (Agrp) hypothalamic neurons to sense and respond to changes in peripheral energy stores. Leptin and insulin have been implicated as circulating indicators of adiposity, but it is not clear how changes in their levels are perceived or integrated by individual neuronal subtypes. We developed mice in which a fluorescent reporter for PI3K activity is targeted to either Agrp or POMC neurons and used 2-photon microscopy to measure dynamic regulation of PI3K by insulin and leptin in brain slices. We show that leptin and insulin act in parallel to stimulate PI3K in POMC neurons but in opposite ways on Agrp neurons. These results suggest a new view of hypothalamic circuitry, in which the effects of leptin and insulin are integrated by anorexigenic but not by orexigenic neurons.
\end{abstract}

\section{Introduction}

Control of energy homeostasis requires the central nervous system to sense and respond to changes in peripheral energy stores. Among first-order neurons suggested to date are those found in the hypothalamic arcuate nucleus that express either proopiomelanocortin (POMC) or agouti-related protein (Agrp), neuropeptides that promote negative and positive energy balance, respectively. The activity of these neurons is regulated in a reciprocal manner by leptin, an adipocyte-derived hormone that conveys afferent input from the periphery to the brain regarding the status of body energy stored in the form of fat (reviewed in refs. 1, 2, 3).

The weight-reducing action of leptin involves both the inhibition of Agrp neurons (which coexpress the potent orexigen neuropeptide Y) and the stimulation of anorexigenic POMC neurons. Conversely, deficient leptin signaling in $o b / o b$ or $d b / d b$ mice activates Agrp neurons while inhibiting POMC neurons, a combination that increases food intake and reduces energy expenditure, causing profound obesity. At least 1 downstream component of leptin receptor activation involves phosphorylation of the transcription factor Stat3, and genetic manipulations that interfere with this process cause obesity (4-6), although the relative importance of Stat 3 activation in different neuronal subtypes has not been addressed. Previous studies of hypothalamic circuitry and energy balance have focused on changes in membrane potential ( 7 , 8 ), activation of Fos (2), or modulation of synaptic plasticity (9). However, the fundamental question of how leptin activates one cell type but inhibits another remains unanswered.

Here we report the development of a molecular genetics strategy that permits dynamic and cell-specific measurement of PI3K activation in hypothalamic POMC or Agrp neurons, using a brain slice

Nonstandard abbreviations used: aCSF, artificial cerebrospinal fluid; Agrp, agouti-related protein; BAC, bacterial artificial chromosome; EGFP, enhanced green fluorescent protein; EGFP-PH(Grp1), fusion protein of EGFP and PH-Grp1; MSH, melanocyte-stimulating hormone; PIP3, phosphatidylinositol triphosphate; POMC, proopiomelanocortin; R26R, Gt(Rosa)26Sortm1Sor; TTX, tetrodotoxin.

Conflict of interest: The authors have declared that no conflict of interest exists.

Citation for this article: J. Clin. Invest. 115:951-958 (2005)

doi:10.1172/JCI200524301. preparation and time-lapse 2-photon microscopy. We focused on $\mathrm{PI} 3 \mathrm{~K}$ as a potential target for leptin action because it plays a central role in transducing external events that rapidly affect behavior in a variety of cell types (10), including hypothalamic neurons (11), and is the primary target of action by insulin, which is thought to function similarly to leptin as a central signal of peripheral energy stores (12-14). Most important, intracerebroventricular administration of PI3K inhibitors blocks the ability of leptin (15) and insulin (13), but not other anorexigenic compounds, to reduce food intake; thus, PI3K is likely to lie downstream of leptin and insulin signaling, either directly or indirectly.

As described below, we find that the effects of leptin and insulin converge on POMC neurons, where each hormone directly activates $\mathrm{PI} 3 \mathrm{~K}$ in the absence of synaptic transmission. By contrast, leptin and insulin have opposite effects on Agrp neurons, an observation that correlates with differences in neuronal wiring such that the effect of insulin on Agrp neurons is direct while the effect of leptin is, surprisingly, indirect. These results provide a new view of humoral adiposity signals and circuitry of their hypothalamic targets.

\section{Results}

Dynamic measurement of PI3K signaling in transgenic mice. To obtain dynamic measurements of PI3K activity in living POMC or Agrp neurons, we employed a fluorescent reporter protein in which enhanced green fluorescent protein (EGFP) is fused to the pleckstrin homology $(\mathrm{PH})$ domain from Grp1 [EGFP$\mathrm{PH}(\mathrm{Grp} 1)$ ], which translocates to the cell membrane upon binding phosphatidylinositol triphosphate (PIP3), the product of PI3K activation (16-18). In cultured cells, the EGFP-PH(Grp1) protein system provides a robust histochemical approach with which to measure PI3K activation in response to insulin (18). To achieve expression of EGFP-PH(Grp1) specific to POMC or Agrp neurons, we constructed an adenoviral vector in which EGFP-PH(Grp1) coding sequences were placed after a strong promoter (cytomegalovirus promoter) and a concatenated polyadenylation site (4xpA) flanked by loxP sites (Figure 2A). After injection of the adenoviral vector into the mediobasal hypothalamus of mice carrying a Cre recombinase transgene driven by regulatory elements from either Pomc 


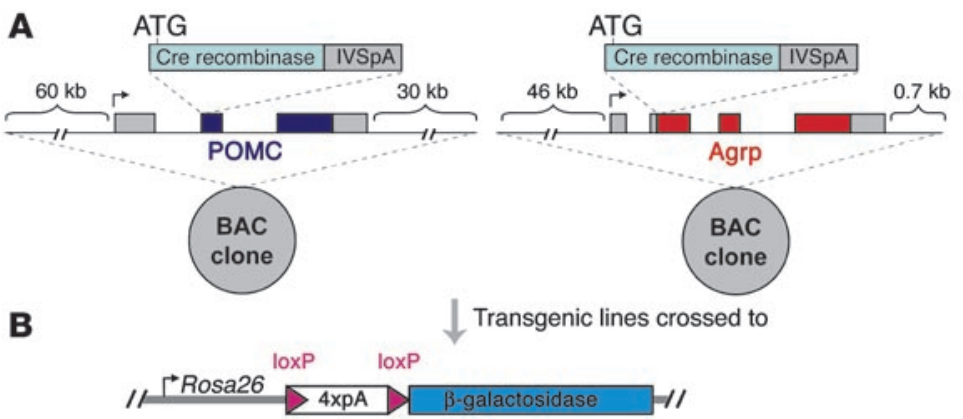

or
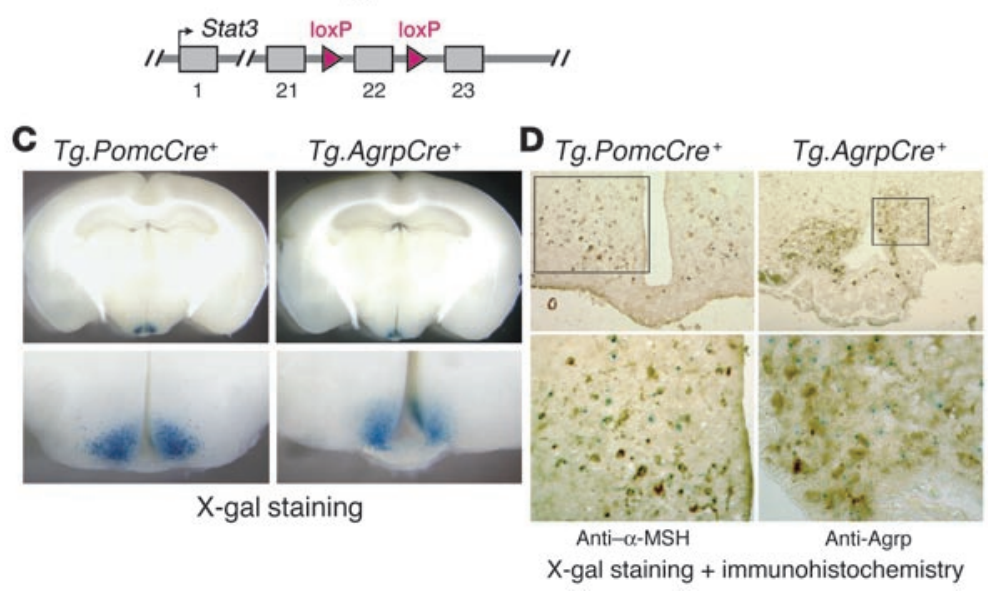

\section{Figure 1}

Generating PomcCre, AgrpCre transgenic mice, and neuron-specific Stat3 knockout mice. (A) Transgene diagrams in which blue and red boxes represent protein-coding regions for the Pomc and Agrp genes, respectively. (B) Schematic representation of the Rosa26 reporter (top) and Stat3 (bottom). (C) X-gal staining of brain sections through the arcuate nucleus from transgenic $R 26 R^{+}$mice. (D) $X$-gal staining (blue) combined with immunohistochemistry (brown) for $\alpha-\mathrm{MSH}$ and Agrp; neuropeptide immunostaining is distributed throughout neuronal cell bodies and also in some terminals; X-gal staining is a localized precipitate within the neuronal cytoplasm.

After injection of the recombinant adenovirus carrying Cre-activatable EGFP-PH(Grp1) into the mediobasal hypothalamus of Tg.PomcCre or Tg.AgrpCre animals, a coronal brain section $(500 \mu \mathrm{m})$ was taken through the arcuate nucleus of the hypothalamus and was placed into an oxygenated perfusion chamber for time-lapse imaging with a 2-photon laser-scanning microscope (Figure 2, B and C). To confirm that EGFP-PH(Grp1) serves as a PI3K reporter protein in hypothalamic POMC or Agrp neurons, we added 1, 10, or $100 \mathrm{nM}$ insulin to the perfusate after a 60 -minute equilibration period. (During this equilibration period in artificial cerebrospinal fluid [aCSF], we occasionally observed initial membrane localization of the PI3K reporter protein in both POMC and Agrp neu-

(Tg.PomcCre) or Agrp (Tg.AgrpCre), the EGFP-PH(Grp1) reporter was activated only in POMC or Agrp neurons due to cell type-specific expression of Cre recombinase (Figure 2, A-C).

Tg.PomcCre and Tg.AgrpCre mice were generated by placing a Cre cDNA cassette into a bacterial artificial chromosome (BAC) clone carrying the Pomc or Agrp genes (Figure 1A) and producing transgenic mice (Figure $1 \mathrm{~B}$ ). Using the lac $Z$ reporter allele Gt(Rosa)26Sor tm1Sor (R26R) to evaluate specificity (19), we identified transgenic lines in which the pattern of $R 26 R$ expression corresponded to the locations of the POMC and Agrp neurons in the lateral and medial portions of the arcuate nucleus, respectively (Figure 1C). Most transgenics exhibited expression in the arcuate nucleus; however, a substantial proportion also exhibited expression in other regions of the brain (Table 1). Based on X-gal staining, we selected transgenic lines that recapitulated the pattern of endogenous POMC and Agrp expression for further study. We used simultaneous X-gal staining and neuropeptide immunohistochemistry to confirm that the majority of lacZ-expressing neurons in these Tg.PomcCre or Tg.AgrpCre lines immunostained for POMC or Agrp neuropeptides, respectively (Figure 1D). rons, but this pattern invariably diffused and the reporter became uniformly distributed during equilibration.) After the equilibration period and the addition of insulin, we observed, as expected, dose- and time-dependent accumulation of a fluorescent signal at discrete locations along the neuronal plasma membrane (Figure $2 D)$. Insulin-induced membrane localization was typically apparent 30-60 seconds after stimulation and often persisted for 10-15 minutes during constant exposure, but then the reporter gradually returned to the cytoplasm. A strong fluorescent signal was also observed in the cell nucleus but was unrelated to insulin treatment or experimental manipulation, as has been reported in studies of cultured cells $(17,18)$. We confirmed that membrane localization of the reporter protein was an indicator of PI3K activation by pretreating the slices with either of 2 pharmacological inhibitors of PI3K (LY294002 or Wortmannin), each of which fully blocked insulin-induced membrane accumulation of the reporter protein (data not shown).

Effects of leptin on PI3K activity in neuronal subsets. To investigate leptin regulation of PI3K signaling in POMC and Agrp neurons, we developed a quantitative approach for measuring membrane

Table 1

Generation of Tg.PomcCre and Tg.AgrpCre transgenic lines

$\begin{array}{lcccc}\text { Transgene } & \text { No. founders } & \text { Expression in arcuate nucleus } & \text { Ectopic expression } & \text { No expression } \\ \text { PomcCre } & 26 & 22 & 14 & 4 \\ \text { AgrpCre } & 6 & 6(2 \text { weak }) & 2 & 0\end{array}$

Expression of the PomcCre or AgrpCre transgene was determined by the pattern of X-gal staining in transgenic animals that also carried the $R 26 R$ reporter gene, as indicated in Figure 1. Ectopic expression refers to the presence of X-gal staining in brain regions outside the arcuate nucleus of the hypothalamus. Entries represent independent transgenic founders, with X-gal staining analyzed either in the founder animal itself or its progeny when established as a transgenic line. 
A

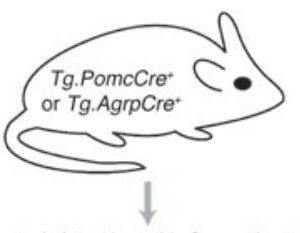

Sterotaxic injection with Cre-activatable EGFP-PH(Grp1) adenovirus

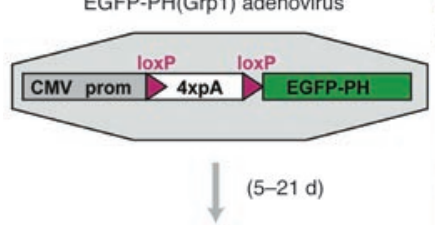

Live brain slice imaged with 2-photon laser microscope

D

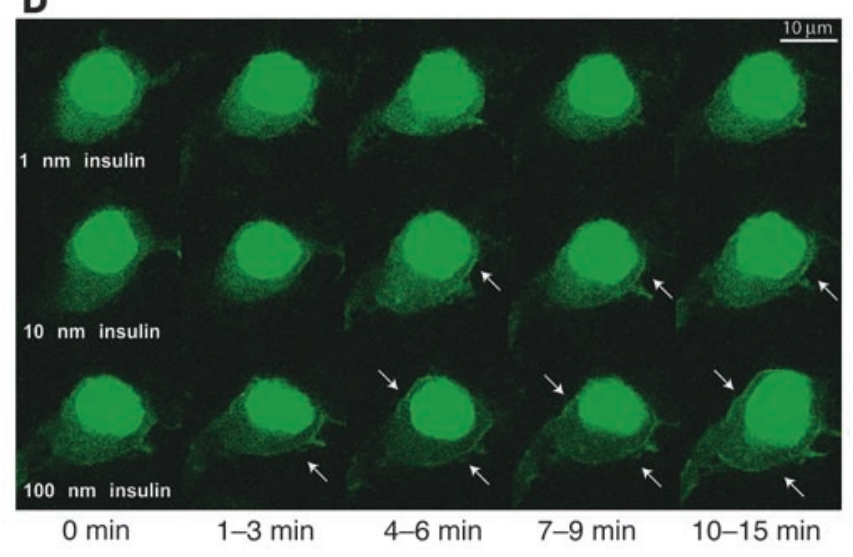

accumulation of the PI3K reporter protein. Optical sections of neurons captured during 2-photon imaging were analyzed with a MatLab edge detection algorithm, and the extent of membrane localization was assessed at each time point by the number of edge pixels per cell membrane.

Using this approach, we found that the addition of $100 \mathrm{nM}$ leptin triggered membrane accumulation of the PI3K reporter protein in 20 of 29 POMC neurons examined. As with insulin, membrane fluorescence triggered during constant exposure to leptin was found in localized patches that varied in different neurons during the course of the experiment (see supplemental material; available online with this article; doi:10.1172/JCI200524301DS1). In some confocal images, membrane fluorescence intensity did not appear as bright as that induced by insulin; however, quantitative measurements demonstrated that leptin-induced membrane localization of the PI3K reporter protein was blocked completely by pretreatment with LY294002 or Wortmannin (Figure 3, A and C). Thus, leptinmediated PI3K activation may contribute to the well-documented stimulatory effect of leptin on POMC neurons $(7,20)$.

In brain slices from $\mathrm{Tg}$.AgrpCre mice, we found that the addition of $100 \mathrm{nM}$ leptin to the perfusate failed to stimulate membrane localization of the PI3K reporter protein. However, because expression of Agrp is activated by conditions characterized by low plasma leptin levels (such as fasting), we hypothesized that in the Agrp neurons, PI3K contributes to the activation of the neurons and is activated by the absence of leptin. To investigate this hypothesis, we preincubated brain slices from Tg.AgrpCre mice in $100 \mathrm{nM}$ leptin during the equilibration period and then removed leptin by

\section{Figure 2}

Imaging system to measure dynamic activation of PI3K in live hypothalamic neurons. (A) Outline of experimental procedure. CMV prom, cytomegalovirus promoter. (B and C) Low- (B) and high-magnification (C) images illustrating adenoviral expression of the PI3K reporter in a Tg.PomcCre+ mouse. (D) Dose-dependent response to insulin of PI3K reporter membrane translocation in POMC neurons. Representative images from various time frames are shown; 0 minutes indicates either before insulin addition or after extensive wash following a previous treatment. Arrows indicate membrane localization.

perfusion with aCSF. Under these conditions, leptin withdrawal triggered membrane accumulation of the PI3K reporter in 18 of 21 Agrp neurons examined (Figure 3B and Figure 4A). Thus, reciprocal regulation of POMC and Agrp neurons by leptin correlates with reciprocal effects on $\mathrm{PI} 3 \mathrm{~K}$ activation.

The majority of POMC or Agrp neurons responded to leptin addition or leptin withdrawal, respectively, within 10 minutes (Figure 3D). However, the duration of the response varied, with some neurons exhibiting transient membrane localization for less than 1 minute and others exhibiting sustained membrane localization for more than 10 minutes. Overall, the timing of PI3K activation in response to a change in leptin levels was similar in POMC and Agrp neurons whether analyzed according to the cumulative distribution of earliest response (Figure 3D) or the proportion of neurons exhibiting a response within a particular time window (Figure 3E).

$P I 3 K$ regulation by insulin, and effects of synaptic inbibitors. As described above, leptin and insulin had similar effects on POMC neurons: addition of either compound caused membrane accumulation of the PI3K reporter protein (Figure 2D and Figure 3). Surprisingly, leptin and insulin had opposite effects on Agrp neurons, with membrane accumulation of the reporter protein inhibited by leptin but stimulated by insulin (Figure 4A). The time course of insulin-induced PI3K activation in Agrp neurons appeared similar to that in POMC neurons, with membrane accumulation evident within a few minutes of hormone addition, then declining gradually over 30-60 minutes (Figure 2D and Figure 4A). Unlike POMC neurons, however, activation of PI3K by insulin in Agrp neurons was the opposite of what would be predicted for a physiologic adiposity signal.

To gain further insight into the mechanisms underlying these effects, we asked whether the PI3K response of POMC and Agrp neurons was altered by inhibitors of synaptic transmission. This was accomplished by incubating brain slices in tetrodotoxin (TTX) or reduced extracellular calcium (which block voltage-gated sodium channels and calcium-dependent synaptic vesicle release, respectively) prior to measuring membrane localization of the PI3K reporter protein in response to changes in leptin or insulin.

Neither TTX nor reduced extracellular calcium affected the response of POMC neurons to leptin addition (Figure 4C), but both treatments markedly reduced the response to leptin withdrawal in Agrp neurons (Figure 4D). Thus, leptin directly activates PI3K in POMC neurons, but the effect of leptin withdrawal in activating PI3K in Agrp neurons requires synaptic transmission and therefore must be mediated indirectly. We also tested whether synaptic transmission was required for the effects of insulin on PI3K activation and found that neither reduced extracellular calcium nor TTX diminished PI3K activation in POMC or Agrp neurons (Figure 4, B, E, and F); in fact, reduced extracellular cal- 
A Tg.PomcCre; treated with $100 \mathrm{nM}$ leptin

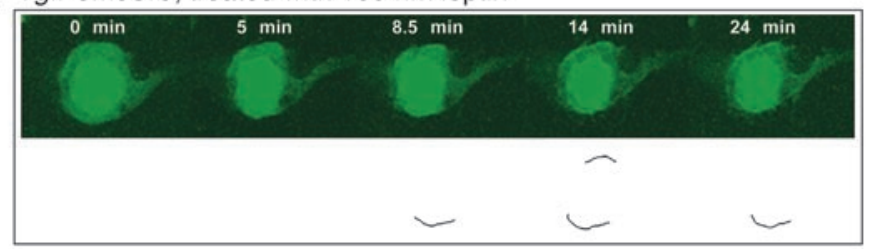

B Tg.AgrpCre; treated with $100 \mathrm{nM}$ leptin pretreatment
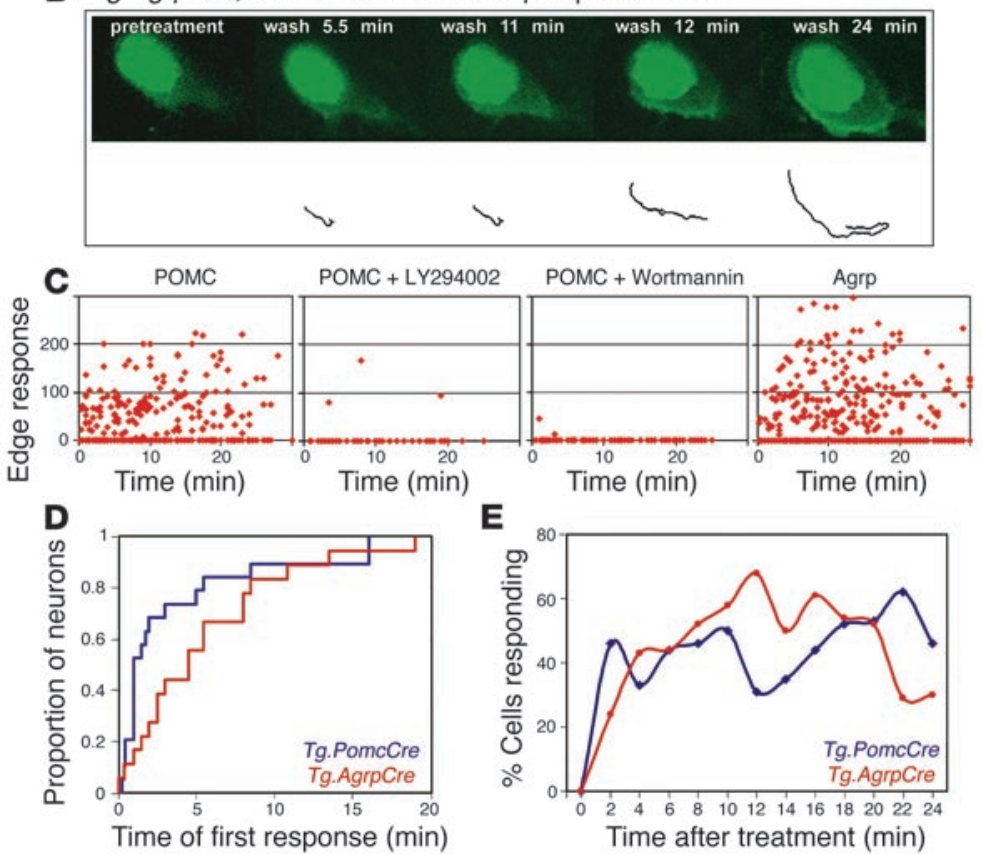

E

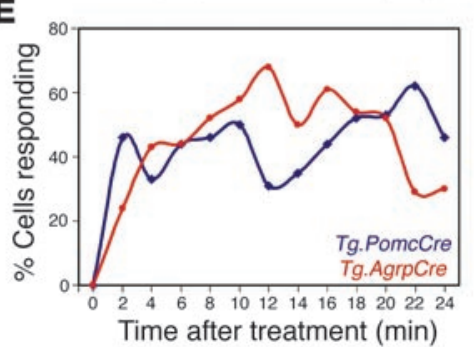

Figure 3

Differential regulation of PI3K by leptin in POMC and Agrp neurons. Example of $\mathrm{PI} 3 \mathrm{~K}$ reporter distribution during leptin treatment of a POMC neuron $(\mathbf{A})$ and leptin withdrawal from an Agrp neuron (B). Upper and lower panels show the raw image and corresponding cell membrane edges, respectively. (C) Distribution of edge pixels as a function of time under the indicated conditions. (D) Cumulative distribution of the earliest time at which responsive POMC $(n=19)$ or Agrp $(n=18)$ neurons first manifest reporter translocation. (E) Percentage of all neurons examined that were responsive to leptin addition (POMC, $n=29$ ) or withdrawal (Agrp, $n=21$ ); each point represents a 2-minute window.

rescent cells would have undergone Cre-mediated excision of Stat3. We found no difference in leptin-induced PI3K activation between POMC-specific Stat3 mutant animals and animals with intact Stat 3 expression, measured as either the proportion of neurons activated (Figure 5, A and B) or the timing of activation (data not shown). Thus, activation of PI3K by leptin in POMC neurons does not require functional Stat3.

\section{Discussion}

Previous electrophysiologic studies have painted an intricate but complex picture of hypothalamic energy balance circuitry. POMC neurons marked with an EGFP transgene are depolarized in response to both leptin (7) and elevated extracellular glucose (20); however, a subset of unmarked hypothalamic neurons selected for the same type of glucose responsiveness was found to be hyperpolarized by leptin (26). Our findings provide

cium caused a small but significant increase in the proportion of POMC neurons that exhibited insulin-induced PI3K activation, possibly due to relief of tonic inhibitory input from adjacent Agrp neurons (7). Taken together, these results suggest a fundamental difference in the way POMC and Agrp neurons modulate PI3K activity in response to leptin, not only in direction but also in the underlying mechanism, with leptin having a primary effect on POMC neurons but a secondary effect on Agrp neurons.

Leptin-induced PI3K activation in POMC neurons is independent of Stat 3 function. The PI3K pathway has received less attention than the JAK-STAT pathway as an effector of leptin action, in part because JAK-STAT signaling is a prototype for type I cytokine receptors and in part because genetic manipulations that interfere with leptininduced Stat 3 phosphorylation cause obesity (4-6). However, Stat 3 acts primarily as a transcription factor $(21,22)$, and leptin regulation of arcuate nucleus neurons clearly involves actions that are independent of changes in gene expression, including regulation of electrical activity and modulation of $\operatorname{PI} 3 \mathrm{~K}(7,8,11,15,23)$.

Because Stat 3 has also been reported as serving as an adapter protein that recruits PI3K to the activated type I interferon receptor (24), we determined whether leptin-induced PI3K activation in POMC neurons requires Stat 3 . We first generated mice in which functional Stat3 is removed specifically from Cre-expressing POMC neurons (Figure 1B), using a previously validated loxPflanked Stat 3 allele (25), and then injected these animals with our adenovirus carrying the Cre-activatable PI3K reporter. Because expression of the PI3K reporter protein serves as a cell-autonomous marker for Cre-mediated recombination events, most fluo- a new perspective with respect to the nature of both the response and the circuitry, in which PI3K may contribute to the activation of both Agrp and POMC neurons, but the direction of its regulation by leptin depends on whether the action is mediated directly or indirectly (Figure 5C). Thus, reciprocal regulation of PI3K by leptin provides a unifying hypothesis to explain how this hormone exerts opposing effects on the activity of these 2 key subsets of neurons.

An important implication of our findings has to do with the notion that insulin and leptin play similar roles as peripheral indicators of energy balance. This idea was initially based on observations that insulin circulates and enters the brain at a level proportional to the body fat stores and has been supported by observations that central administration of insulin inhibits food intake and reduces body weight (reviewed in refs. 1, 14) and that neuron-selective insulin receptor knockout mice develop moderate diet-induced obesity (27).

Our results suggest that parallel effects of leptin and insulin on energy balance could be integrated at the level of POMC neurons, where both hormones activate PI3K, and leptin has been shown to cause membrane depolarization and an increased firing rate. Although previous studies by Ashford and colleagues $(11,26)$ identified a subset of hypothalamic neurons in which the effects of leptin or insulin on membrane hyperpolarization could be blocked by PI3K inhibitors, our results do not distinguish whether changes in POMC PI3K are independent of or causally related to changes in POMC electrical activity. Both phenomenon occur rapidly and may contribute to acute responses such as neuropeptide release and rapid changes in feeding behavior. 

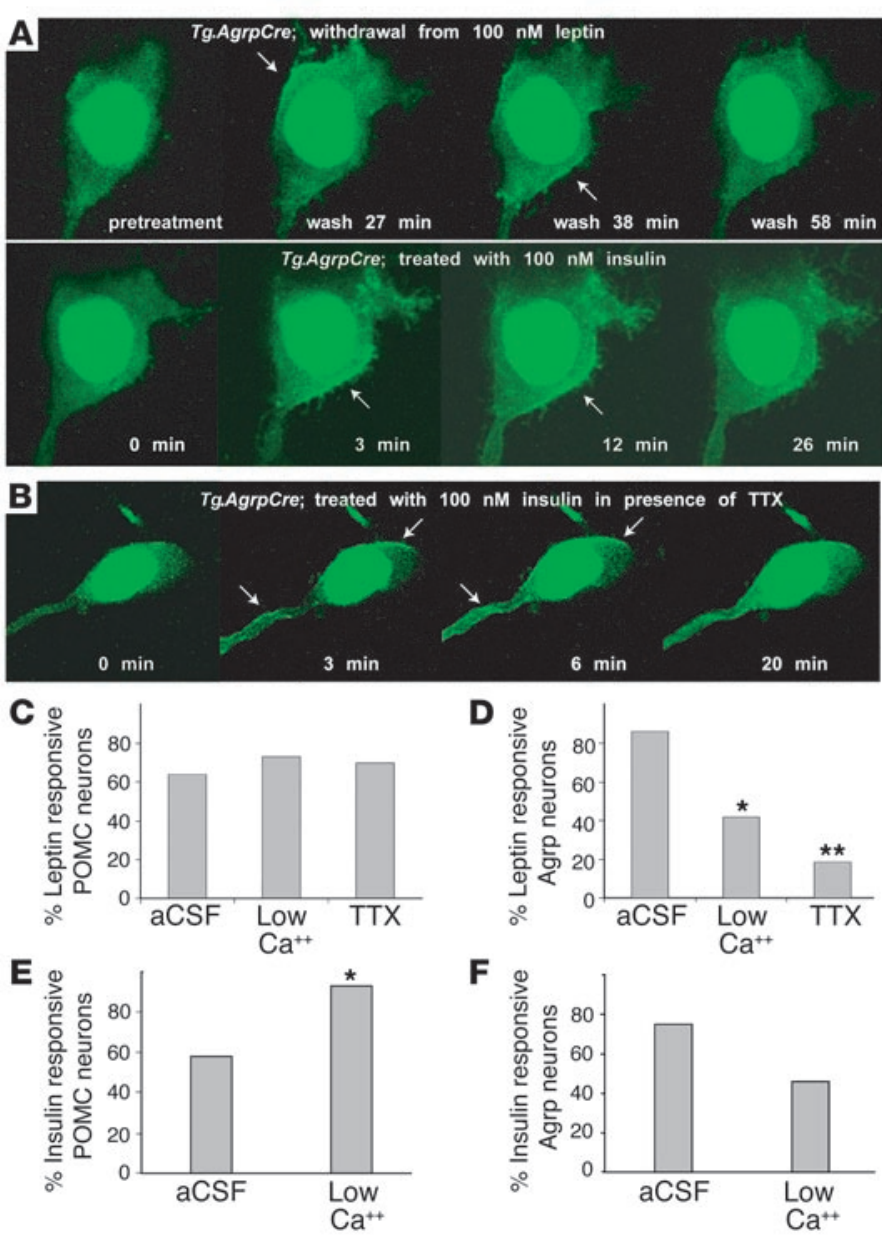

However, our observation that insulin and leptin have opposite effects on PI3K activity in Agrp neurons suggests that the functions of these 2 hormones as energy balance signals do not overlap completely. Indeed, differential effects of leptin and insulin on Agrp expression have been described previously in the setting of streptozotocin-induced diabetes, in which leptin, but not insulin administration, normalizes Agrp expression $(28,29)$. Furthermore, the effects of insulin on neuronal growth and survival exceed what might be expected for a simple adiposity signal, with widespread

\section{Figure 5}

$\mathrm{PI} 3 \mathrm{~K}$ activation in POMC neurons does not require Stat3 function. (A) Representative Stat3-deficient POMC neuron exposed to $100 \mathrm{nM}$ leptin, in which membrane localization of PI3K reporter protein occurs within several minutes of hormone addition. Arrows indicate membrane localization. (B) Percentage of POMC neurons responsive to leptin in the presence $(+/+)$ or absence $(-/-)$ of Stat3. (C) Unifying mechanism for leptin modulation of key arcuate nucleus neurons in which PI3K activity is a mediator and/or marker of neuronal activation and neuropeptide release in both Agrp (pink) and POMC (green) neurons. The effects of insulin on PI3K activity are direct in both neuronal subtypes, but the effects of leptin on PI3K activity in Agrp neurons require synaptic transmission from POMC or other (gray) inhibitory presynaptic neurons. IR, insulin receptor; LepR, leptin receptor.

\section{Figure 4}

PI3K regulation by insulin in Agrp neurons and effects of synaptic inhibitors in POMC and Agrp neurons. (A) In Agrp neurons, leptin withdrawal has the same effect as insulin addition; both treatments stimulate membrane localization of the PI3K reporter protein. The example shown represents the same neuron in which a slice preincubated with $100 \mathrm{nM}$ leptin for 60 minutes was then perfused with aCSF; during this time the PI3K reporter protein exhibited initial membrane localization followed by a return to the cytoplasm by 58 minutes. Addition of $100 \mathrm{nM}$ insulin then triggered membrane localization again. In other experiments, $100 \mathrm{nM}$ insulin was observed to cause PI3K activation in Agrp neurons that had not previously been exposed to leptin. Arrows indicate membrane localization. (B) Example of the same experiment as in $\mathbf{A}$ but carried out in the presence of $1 \mu \mathrm{M}$ TTX (which blocked the previous response to leptin withdrawal). (C and D) Percentage of POMC (C) and Agrp (D) neurons responsive to leptin with (aCSF) or without (low $\mathrm{Ca}^{++}$or TTX) synaptic transmission. ${ }^{*} P<0.05$; ${ }^{\star *} P<0.01$ as determined by $\chi^{2}$ analysis. ( $E$ and $\mathbf{F})$ Percentage of POMC (E) and Agrp (F) neurons responsive to insulin with (aCSF) or without (low $\mathrm{Ca}^{++}$) synaptic transmission.

expression of insulin receptors in many brain regions (30) and a connection between insulin action and neurodegeneration $(31,32)$. Finally, leptin and the melanocortin system are specific to vertebrates while the effects of insulin on the brain are conserved across all metazoans, with genetic studies in invertebrates pointing to an ancient role for insulin in energy balance as well as aging and growth $(33,34)$. From this perspective, leptin may serve as a specialized energy balance signal invented during vertebrate evolution whose effects partially overlap with a more ancient role for insulin.

In addition to the differential effects of leptin on PI3K activity in POMC and Agrp neurons, our results indicate that the circuits underlying these effects also differ, since the inhibitory effect of leptin on PI3K in the majority of Agrp neurons requires synaptic transmission. However, previous neuroanatomical studies by us and by others have demonstrated that a subset of Agrp neurons (or Npy neurons in the arcuate nucleus) expresses the long form of the leptin receptor (35-39). This apparent paradox - an indirect response to leptin despite the presence of leptin receptors on some Agrp neurons - could be explained if Agrp neurons require multiple signals to activate $\mathrm{PI} 3 \mathrm{~K}$ in response to leptin withdrawal, some that are mediated autonomously via a leptin receptor and others that are mediated via synaptic trans-

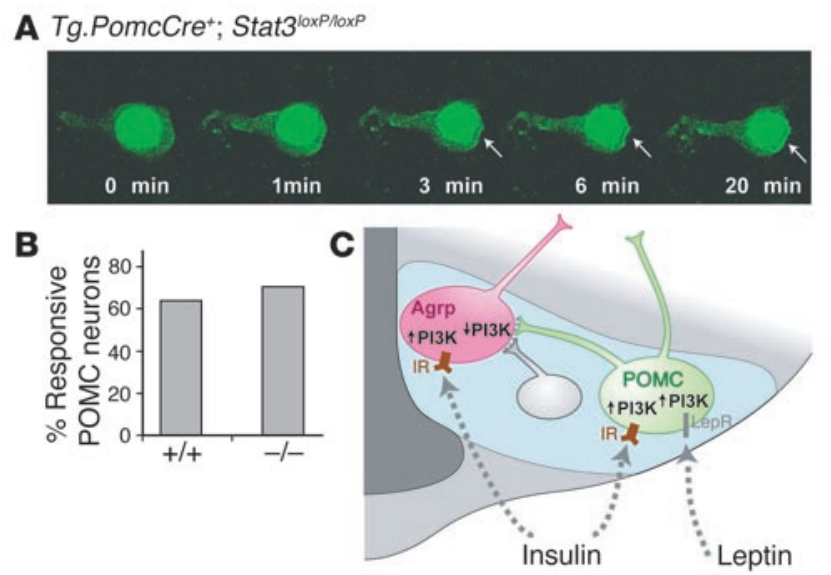


mission. Alternatively, there may be functional heterogeneity within Agrp neurons if, for example, the subset of Agrp neurons that express the leptin receptor are distinct from those that activate PI3K in response to leptin withdrawal. If so, this latter group of neurons might receive leptin-mediated inhibitory signals from POMC or other neurons via $\gamma$-aminobutyric acid (GABA) (40) or other inhibitory neurotransmitters (Figure 5C).

Heterogeneity of POMC neurons might also help to explain the observation that leptin activation of PI3K in POMC neurons does not require Stat 3 phosphorylation. A recent neuroanatomical study reported that $37 \%$ of POMC cells were immunopositive for phospho-Stat 3 after leptin treatment (41), and in our imaging system, leptin activated PI3K in approximately $60 \%$ of the POMC neurons. Thus, leptin receptor engagement on distinct subsets of POMC neurons could activate different downstream effectors. Regardless, these results together with earlier work $(7,42)$ suggest a view of leptin signaling in which there are 2 types of direct intracellular effectors with different actions and possibly different mechanisms of activation. One type, exemplified by Stat 3 , is likely to mediate responses that require changes in gene expression, whereas a second type, exemplified by the direct effects of leptin on PI3K activation and electrical activity in POMC neurons, is likely to mediate acute responses such as neuropeptide release and rapid changes in feeding behavior. In contrast to Stat3, whose mechanism of activation by leptin receptor engagement is well characterized $(21,42,43)$, the biochemical mechanisms by which leptin activates PI3K and modulates membrane potential in POMC neurons are not yet clear. However, the two may be causally related, since depolarization of POMC neurons by leptin involves a nonselective cation channel (7) and PIP3 has recently been shown to be capable of modulating ion channels $(44,45)$. In addition, work of Myers and colleagues (6) has shown that animals carrying a modified leptin receptor that cannot activate Stat3, Lepr ${ }^{1138 S e r}$, develop a subset of the same phenotypic abnormalities caused by a null Lepr allele; thus, it will be interesting to examine the effects of leptin on PI3K activation and electrical activity in POMC neurons that carry the Lepr ${ }^{1138 s e r}$ mutation.

We chose to focus on POMC and Agrp neurons because of previous work suggesting they serve as primary sensors for circulating adiposity signals. Balthasar et al. (46) recently demonstrated that animals with POMC-specific Lepr deletion develop a mild obesity and metabolic derangement. To the extent that results from different studies can be compared, the effect of the POMCspecific Lepr deletion appears to be less dramatic than that of a complete Pomc, Mc4r, or Lepr deletion (47). These observations suggest that POMC neurons use mediators and/or signaling mechanisms in addition to leptin to control body weight and, furthermore, that the effect of leptin on CNS control of body weight involves neurons in addition to those that express Pomc. In this regard, comparison of POMC-specific Lepr deletion mice with the POMC-specific Stat 3 deletion mice could reveal the extent to which leptin signaling in POMC neurons is mediated by Stat 3 or other effectors such as PI3K.

The approach introduced here - a combination of transgenic biology and gene therapy that enables dynamic histochemical measurements of neuronal cell signaling recorded by multiphoton microscopy of brain slices - can be applied to additional signaling events and to diverse pathophysiologic problems in which the brain measures and responds to changes in environmental parameters including $\mathrm{pH}$, temperature, glucose concentration, and cytokine levels. Advances in image processing and miniaturization should allow events from multiple neurons to be recorded simultaneously from living animals, an advance that could help in the dissection of molecular and cellular events that underlie complex behaviors.

\section{Methods}

Recombineering and generation of transgenic mice. The BAC we used, clone 389J9 (Research Genetics Inc.), contained approximately $60 \mathrm{~kb}$ of $5^{\prime}$ and approximately $30 \mathrm{~kb}$ of $3^{\prime}$ flanking sequence and was modified by homologous recombination in bacteria using the 2-step RecE and RecT-mediated recombination (ET cloning) method of Stewart and colleagues (48), in which a neomycin resistance cassette flanked by FLP recombinase target sites was used for positive selection and then excised by transient expression of FLP recombinase. Cre recombinase coding sequences modified to contain an optimal translational initiation site and nuclear localization signal were derived from a plasmid originally constructed by M. Lewandoski (National Cancer Institute - Frederick Cancer Research and Development Center, Frederick, Maryland, USA), pML78. A $1.1 \mathrm{~kb}$ SalI-EcoRI fragment from pML78 that includes approximately $35 \mathrm{nt}$ of $5^{\prime}$ untranslated sequence and approximately $20 \mathrm{nt}$ of $3^{\prime}$ untranslated sequence was fused to a $0.6 \mathrm{~kb}$ EcoRI fragment from the mouse protamine 1 gene that contains an intron and polyadenylation signal (49), and this CreIVSpA fragment was then placed upstream of an FLPflanked neomycin resistance cassette. We placed $50 \mathrm{bp}$ Pomc homology arms at both ends of this cassette, electroporated the linear fragment into bacteria carrying BAC clone 389J9, screened neomycin-resistant clones by PCR and restriction digest analysis for correct targeting, and then removed the neomycin cassette with FLP recombinase. The homology arms were chosen so as to insert the CreIVSpA cassette within exon 2 precisely upstream of the normal Pomc translational initiation site; the $5^{\prime}$ and $3^{\prime}$ homology arms correspond to residues 102-151 and 152-201, respectively, from the Pomc cDNA (GenBank accession number NM_00889) - the normal Pomc translational initiation site lies at residue 152-154.

For Agrp, we identified a BAC from a C57BL/6J library (Genome Systems Inc.), clone $171 \mathrm{n} 11$, that carried approximately $67 \mathrm{~kb}$ of $5^{\prime}$ and approximately $37 \mathrm{~kb}$ of $3^{\prime}$ flanking sequence. Using the same strategy as for Pomc, we targeted the CreIVSpA cassette precisely upstream of the normal Agrp translational initiation site in exon 2; the $5^{\prime}$ and $3^{\prime}$ homology arms correspond to residues 107088027-107087978 and 107087977-107087928 in the mouse genome sequence of chromosome 8 (mm4, Oct 2003, National Center for Biotechnology Information [NCBI] build 32) (Agrp is encoded in a reverse orientation with a translational initiation site at residues 107087977-107087975).

For both Pomc and Agrp BACs, linear fragments were purified for microinjection since the BAC vector backbone, pBeloBac11, contains a loxP site. For Pomc, the entire insert was released by digestion with restriction enzyme NotI and purified as an approximately $95 \mathrm{~kb}$ fragment using a 20-35\% sucrose gradient. For Agrp, there are 2 internal Not sites, and we purified a 48.5 $\mathrm{kb}$ NotI fragment using a $20-30 \%$ sucrose gradient that contains approximately $47 \mathrm{~kb}$ of $5^{\prime}$ and approximately $0.7 \mathrm{~kb}$ of $3^{\prime}$ flanking sequence. Linear BAC fragments recovered after sucrose gradient purification were bufferexchanged with microinjection buffer $(10 \mathrm{mM}$ Tris-HCl, $0.1 \mathrm{mM}$ EDTA, $\mathrm{pH}$ 7.4) and concentrated using centriprep- 30 columns before microinjection (at a concentration of $0.6 \mu \mathrm{g} / \mathrm{ml}$ ) into 1-cell embryos produced by mating males homozygous for the $R 26 R$ allele with standard mouse inbred strain $\mathrm{FVB} / \mathrm{N}$ females. Transgenic founders were identified and confirmed by PCR and Southern blot hybridization. We found that 22 of 26 Tg.PomcCre lines and 4 of $6 \mathrm{Tg}$.AgrpCre lines expressed Cre recombinase in the arcuate nucleus of the hypothalamus. In addition, approximately $30 \%$ of animals carrying the AgrpCre transgene exhibited early embryonic expression of Cre recombinase as determined by widespread X-gal staining of multiple somatic tissues; these animals were not used for further experiments. 
$X$-gal staining and immunohistochemistry. For X-gal staining of brain slices, $500 \mu \mathrm{m}$ coronal sections were prepared from freshly dissected brains using an adult brain matrix (Kent Scientific), fixed with $4 \%$ paraformaldehyde in $100 \mathrm{mM}$ PBS and $2 \mathrm{mM} \mathrm{MgCl}_{2}$ (pH 7.4) for 1 hour at $4{ }^{\circ} \mathrm{C}$, then washed 3 times, 10 minutes each time, with PBS containing $0.1 \%$ Triton X-100 at room temperature. Staining was carried out overnight at $37^{\circ} \mathrm{C}$ in PBS that contained $5 \mathrm{mM}$ potassium ferricyanide, $5 \mathrm{mM}$ potassium ferrocyanide, $2 \mathrm{mM} \mathrm{MgCl}_{2}$, and $1 \mathrm{mg} / \mathrm{ml} \mathrm{X}$-gal.

For colocalization studies, mice carrying the R26R allele and either a PomcCre or AgrpCre transgene were first injected with $1 \mu$ l of colchicine $(20 \mu \mathrm{g} / \mu \mathrm{l})$ into the third ventricle, then anesthetized and perfusion-fixed with $4 \%$ paraformaldehyde in $100 \mathrm{mM}$ PBS 48 hours later. Brains were removed, placed in fixative overnight, and infiltrated with $30 \%$ sucrose in PBS at $4^{\circ} \mathrm{C}$, and then $10 \mu \mathrm{m}$ frozen sections were prepared and mounted on Superfrost/Plus slides (Fisher). Sections were washed with $0.1 \%$ Triton $\mathrm{X}-100$ in PBS (3 times, 10 minutes each time), then incubated with X-gal staining buffer (see above) overnight at $37^{\circ} \mathrm{C}$. After X-gal staining, sections were washed with $0.25 \%$ Triton X-100 in PBS ( 3 times, 10 minutes each time), incubated in $0.3 \%$ hydrogen peroxide and $0.3 \%$ normal goat serum in PBS for 5 minutes at room temperature, washed in $0.25 \%$ Triton in PBS, then incubated in $10 \%$ normal goat serum and $1 \%$ BSA in PBS for 1 hour at room temperature. Sections were subsequently immunostained with a rabbit polyclonal $\alpha$-melanocyte-stimulating hormone ( $\alpha$-MSH) antibody (ImmunoStar) at a 1:500 dilution in blocking buffer, or a rabbit polyclonal AGRP antibody raised against human AGRP (35) at a 1:1000 dilution for 1 hour at room temperature. Sections were washed with $0.25 \%$ Triton X-100 in PBS (3 times, 10 minutes each time), then incubated with biotinylated goat-anti-rabbit secondary antibody (Jackson ImmunoResearch Laboratories Inc.) at 1:500 dilution in blocking buffer for 1 hour at room temperature. After washing with $0.25 \%$ Triton in PBS (3 times, 10 minutes each time), we detected biotin using a horseradish peroxidase detection kit (Vector Laboratories).

Cell boundaries of immunostained Agrp neurons were difficult to assess due to the high density of cell bodies and neuronal fibers in the medial portion of the arcuate nucleus, which prevented a quantitative estimate for colocalization. However, results from a different study using the same AgrpCre transgenic line indicated that Cre recombinase was expressed in the majority of Agrp neurons (A.W. Xu and G.S. Barsh, unpublished data). Immunostained POMC neurons were distributed in a manner such that the boundaries of individual cells were readily apparent; in a sample of multiple sections from Tg.PomcCre mice (594 cells counted), 79.5\% of the cells were doubly positive for X-gal staining and MSH immunoreactivity, $18.4 \%$ of the cells were MSH-immunoreactive but did not exhibit X-gal staining, and $2 \%$ of the cells were X-gal positive but not MSH-immunoreactive. Because of the nature of the $\mathrm{R} 26 \mathrm{R}$ reporter, lacZ expression provides information about the history and cell lineage of a neuron. Thus, the very small percentage of X-gal positive but MSH negative neurons may include cells that expressed MSH in the past or in progenitor cells.

Mouse genetics. Animals carrying the R26R lacZ reporter allele were obtained from P. Soriano (University of Washington, Seattle, Washington, USA) and are maintained in our laboratory as homozygotes on a mixed background that includes contributions from FVB/N, C57BL/6J, and 129 strains. The Tg.PomcCre and Tg.AgrpCre lines were maintained as heterozygotes on the same mixed background. Construction and application of the Stat 3 conditional (Stat3 flox $^{\text {(25) }}$ and null (Stat ${ }^{\text {null }}$ ) (50) alleles have been described previously. In brief, the loxP sites flank exon 22, which encodes a phosphorylation site that is required for normal Stat 3 function.

Animals with POMC-specific Stat 3 deletion were generated by intercrossing Tg.PomcCre ${ }^{+}$and Stat $3^{\text {flox } / \text { null }}$ mice. The 4 possible Stat 3 alleles that result from this cross $\left(\right.$ Stat $3^{+}$, Stat $^{\text {null }}$, Stat $3^{\text {flox }}$, and Stat $\left.3^{4}\right)$ were distinguished by
Southern blotting (25); either Stat $3^{\text {flox/null }}$ or Stat $3^{\text {flox/flox }}$ animals were used for imaging studies. The experiments in this study were approved by the Stanford Administrative Panel on Laboratory Animal Care.

Adenoviral vector construction and production. A cDNA encoding EGFP$\mathrm{PH}(\mathrm{Grp} 1$ ) was derived from a plasmid provided by J. Pessin (University of Iowa, Iowa City, Iowa, USA) (18), placed behind a loxP-flanked polyadenylation cassette (4xpA) derived originally from pROSA26-R (19), and then subcloned into the BgIII and HindIII sites of the pShuttle-cytomegalovirus vector (51). This vector was used to produce recombinant adenovirus using the bacteria-based AdEasy system as previously described (51) and high-titer virus $\left(4.2 \times 10^{12} \mathrm{pfu} / \mathrm{ml}\right)$ produced by C. Rhodes (Adenovirus Vector Core, Pacific Northwest Research Institute, Seattle, Washington, USA).

Stereotaxic injection. Injections were carried out on 2- to 4-month-old anesthetized animals using a high precision stereotaxic apparatus (SAS75; Cartesian Research). Coordinates for injection into the arcuate nucleus were $-2.2 \mathrm{~mm}$ bregma, $0.15 \mathrm{~mm}$ lateral, and -5.5 to -5.8 dorsal-ventral. A 1- $\mu 1,25$-gauge Hamilton syringe was used for the injection, and a total volume of $1 \mu \mathrm{l}$ was injected into the arcuate nucleus at a rate of $1 \mathrm{nl} /$ second using a WPI Ump II MicroInjector Unit (Cartesian Research). The needle was left in place for 10 minutes before withdrawal.

Two-photon laser-scanning microscopy. At 5-21 days after stereotaxic injection of the adenovirus, animals were euthanized, and whole brains were removed and each placed in aCSF consisting of $119 \mathrm{mM} \mathrm{NaCl}, 2.5 \mathrm{mM}$ $\mathrm{KCl}, 1 \mathrm{mM} \mathrm{CaCl}, 3 \mathrm{mM} \mathrm{MgCl}, 1 \mathrm{mM} \mathrm{NaH} \mathrm{PO}_{4}, 11 \mathrm{mM}$ glucose, and $26.2 \mathrm{mM} \mathrm{NaHCO}_{3}, \mathrm{pH}$ 7.4). Coronal sections of $500 \mu \mathrm{m}$ through the hypothalamus were immediately cut on ice using a prechilled adult mouse brain matrix (Kent Scientific). Brain slices containing the arcuate nucleus were placed in a perfusion chamber (RC-29 slice chamber; Warner Instruments) that allows for solution to flow to both the top and bottom of the preparation. The chamber was mounted onto a heated stage and imaging carried out on a Zeiss LSM510 confocal laser-scanning microscope maintained by the Stanford Cell Sciences Imaging Facility. Brain slices were constantly perfused with prewarmed $\left(37^{\circ} \mathrm{C}\right) \mathrm{aCSF}$ oxygenated with $95 \% \mathrm{O}_{2}, 5 \% \mathrm{CO}_{2}$ at a rate of $1.2 \mathrm{ml} / \mathrm{min}$. A $\times 40$ magnification water immersion objective (Achroplan IR 40×/0.80 [Zeiss], working distance $3.60 \mathrm{~mm}$ ) and a $\times 63$ magnification water immersion objective (Achroplan IR 63×/0.90 [Zeiss], working distance $2.20 \mathrm{~mm}$ ) were used for imaging. The EGFP-PH(Grp1) fluorescent reporter was excited with a Coherent Mira 900 tunable Ti:Sapphire 2-photon laser at $860-880 \mathrm{~nm}$ (Coherent Inc.) and emission collected by a nondescanned detector using a BP500-550 filter. Twelve-bit images were recorded at a frame size of $1024 \times 1024$ pixels and a rate of $6.40 \mu \mathrm{s} /$ pixel.

In all cases, brain slices were equilibrated for 1 hour in aCSF, during which no spontaneous membrane translocation was observed. To examine the effects of insulin (1nM-100 nM; Sigma-Aldrich) or leptin (100 nM; R\&D Systems) on membrane localization of the fluorescent reporter, we added the hormone directly to the perfusion buffer after equilibration, and images were recorded at intervals of approximately 30 seconds, beginning with the time at which the hormone entered the perfusion chamber. In a few experiments with Tg.PomcCre mice, we tried lower levels of leptin and found that 4 of 7 neurons responded to $25 \mathrm{nM}$ leptin, and 4 of 9 neurons responded to $50 \mathrm{nM}$ leptin. To test whether membrane localization of the reporter protein was dependent on PI3K, we used Wortmannin (100 nM; Sigma-Aldrich) or LY 294002 (10 $\mu \mathrm{m}$; Calbiochem), which were added to the perfusion buffer 30-60 minutes before hormone treatment. To examine the requirement for synaptic transmission, we used low calcium (low $\mathrm{Ca}^{++}, 0.3 \mathrm{mM} \mathrm{CaCl} 2$ ), high magnesium ( $9 \mathrm{mM} \mathrm{MgCl}_{2}$ ), or TTX (1 $\mu \mathrm{M}$; Sigma-Aldrich), which were included at the beginning of the equilibration period. For POMC, 29, 15, 13, and 13 neurons were examined with aCSF, low $\mathrm{Ca}^{++}$, TTX, and in POMCspecific Stat3 deletion mice, respectively; for Agrp, 21, 12, and 16 neurons were examined with aCSF, low $\mathrm{Ca}^{++}$, and TTX, respectively. 
Data analysis. Image files generated during laser scanning were converted into a TIFF format and then analyzed with the Canny edge detection algorithm in MatLab (Version 6.5, release 13). For each series of images, a threshold for edge detection was chosen empirically so that there were no edges detected on the cytoplasmic membrane in images taken prior to hormone treatment. Each image was then edited to remove edges surrounding the nuclear membrane and imported back into MatLab to determine the total number of edge pixels.

\section{Acknowledgments}

We thank J. Mulholland and K. Lee for assistance with imaging; S. Smith and K. Niswender for advice; and C. Rhodes, J. Pessin, and B. Lowell for reagents. We thank N.G. Larsson for providing the Tfam ${ }^{\text {flox/flox }}$ mice. This work was supported by NIH grants DK48506 (to G.S. Barsh) and DK-68384 (to M.W. Schwartz) and by the Stanford Bio-X Interdisciplinary Initiatives Program.

Received for publication December 22, 2004, and accepted in revised form January 25, 2005.

Address correspondence to: Gregory S. Barsh, Departments of Genetics and Pediatrics, Stanford University School of Medicine, Stanford, California, USA. Phone: (650) 723-5035; Fax: (650) 7231399; E-mail: gbarsh@cmgm.stanford.edu.
1. Seeley, R.J., and Woods, S.C. 2003. Monitoring of stored and available fuel by the CNS: implications for obesity. Nat. Rev. Neurosci. 4:901-909.

2. Elias, C.F., et al. 1999. Leptin differentially regulates NPY and POMC neurons projecting to the lateral hypothalamic area. Neuron. 23:775-786.

3. Barsh, G.S., and Schwartz, M.W. 2002. Genetic approaches to studying energy balance: perception and integration. Nat. Rev. Genet. 3:589-600.

4. Gao, Q., et al. 2004. Disruption of neural signal transducer and activator of transcription 3 causes obesity, diabetes, infertility, and thermal dysregulation. Proc. Natl. Acad. Sci. U. S. A. 101:4661-4666.

5. Cui, Y., et al. 2004. Essential role of STAT3 in body weight and glucose homeostasis. Mol. Cell. Biol. 24:258-269.

6. Bates, S.H., et al. 2003. STAT3 signalling is required for leptin regulation of energy balance but not reproduction. Nature. 421:856-859.

7. Cowley, M.A., et al. 2001. Leptin activates anorexigenic POMC neurons through a neural network in the arcuate nucleus. Nature. 411:480-484.

8. Van Den Top, M., Lee, K., Whyment, A.D., Blanks, A.M., and Spanswick, D. 2004. Orexigen-sensitive NPY/AgRP pacemaker neurons in the hypothalamic arcuate nucleus. Nat. Neurosci. 7:493-494.

9. Pinto, S., et al. 2004. Rapid rewiring of arcuate nucleus feeding circuits by leptin. Science. 304:110-115.

10. Cantley, L.C. 2002. The phosphoinositide 3-kinase pathway. Science. 296:1655-1657.

11. Spanswick, D., Smith, M.A., Mirshamsi, S., Routh, V.H., and Ashford, M.L. 2000. Insulin activates ATP-sensitive $\mathrm{K}+$ channels in hypothalamic neurons of lean, but not obese rats. Nat. Neurosci. 3:757-758.

12. Woods, S.C., Lotter, E.C., McKay, L.D., and Porte, D., Jr. 1979. Chronic intracerebroventricular infusion of insulin reduces food intake and body weight of baboons. Nature. 282:503-505.

13. Niswender, K.D., et al. 2003. Insulin activation of phosphatidylinositol 3-kinase in the hypothalamic arcuate nucleus: a key mediator of insulin-induced anorexia. Diabetes. 52:227-231.

14. Schwartz, M.W., Woods, S.C., Porte, D., Jr., Seeley, R.J., and Baskin, D.G. 2000. Central nervous system control of food intake. Nature. 404:661-671.

15. Niswender, K.D., et al. 2001. Key enzyme in leptininduced anorexia. Nature. 413:794-795.

16. Klarlund, J.K., et al. 1997. Signaling by phosphoinositide-3,4,5-trisphosphate through proteins containing pleckstrin and Sec7 homology domains. Science. 275:1927-1930.

17. Kavran, J.M., et al. 1998. Specificity and promiscuity in phosphoinositide binding by pleckstrin homology domains. J. Biol. Chem. 273:30497-30508.

18. Yang, C., Watson, R.T., Elmendorf, J.S., Sacks, D.B., and Pessin, J.E. 2000. Calmodulin antagonists inhibit insulin-stimulated GLUT4 (glucose transporter 4) translocation by preventing the formation of phosphatidylinositol 3,4,5-trisphosphate in 3T3L1 adipocytes. Mol. Endocrinol. 14:317-326.

19. Soriano, P. 1999. Generalized lacZ expression with the ROSA26 Cre reporter strain. Nat. Genet. 21:70-71.

20. Ibrahim, N., et al. 2003. Hypothalamic proopiomelanocortin neurons are glucose responsive and express K(ATP) channels. Endocrinology. 144:1331-1340.

21. Vaisse, C., et al. 1996. Leptin activation of Stat3 in the hypothalamus of wild-type and ob/ob mice but not db/db mice. Nat. Genet. 14:95-97.

22. Myers, M.G., Jr. 2004. Leptin receptor signaling and the regulation of mammalian physiology. Recent Prog. Horm. Res. 59:287-304.

23. Zhao, A.Z., Huan, J.N., Gupta, S., Pal, R., and Sahu, A. 2002. A phosphatidylinositol 3-kinase phosphodiesterase 3B-cyclic AMP pathway in hypothalamic action of leptin on feeding. Nat. Neurosci. 5:727-728.

24. Pfeffer, L.M., et al. 1997. STAT3 as an adapter to couple phosphatidylinositol 3-kinase to the IFNAR 1 chain of the type I interferon receptor. Science. 276:1418-1420.

25. Takeda, K., et al. 1998. Stat3 activation is responsible for IL-6-dependent T cell proliferation through preventing apoptosis: generation and characterization of T cell-specific Stat3-deficient mice. J. Immunol. 161:4652-4660.

26. Spanswick, D., Smith, M.A., Groppi, V.E., Logan, S.D., and Ashford, M.L. 1997. Leptin inhibits hypothalamic neurons by activation of ATP-sensitive potassium channels. Nature. 390:521-525.

27. Bruning, J.C., et al. 2000. Role of brain insulin receptor in control of body weight and reproduction. Science. 289:2122-2125.

28. Havel, P.J., et al. 2000. Effects of streptozotocininduced diabetes and insulin treatment on the hypothalamic melanocortin system and muscle uncoupling protein 3 expression in rats. Diabetes. 49:244-252.

29. Hidaka, S., et al. 2001. Hypoleptinemia, but not hypoinsulinemia, induces hyperphagia in streptozotocin-induced diabetic rats. J. Neurochem. 77:993-1000

30. Havrankova, J., Roth, J., and Brownstein, M. 1978. Insulin receptors are widely distributed in the central nervous system of the rat. Nature. 272:827-829.

31. Gasparini, L., Netzer, W.J., Greengard, P., and $\mathrm{Xu}$, H. 2002. Does insulin dysfunction play a role in Alzheimer's disease? Trends Pharmacol. Sci. 23:288-293.

32. Schubert, M., et al. 2003. Insulin receptor substrate2 deficiency impairs brain growth and promotes tau phosphorylation. J. Neurosci. 23:7084-7092.

33. Rulifson, E.J., Kim, S.K., and Nusse, R. 2002. Ablation of insulin-producing neurons in flies: growth and diabetic phenotypes. Science. 296:1118-1120.

34. Wolkow, C.A., Kimura, K.D., Lee, M.S., and Ruvkun, G. 2000. Regulation of C. elegans life-span by insulinlike signaling in the nervous system. Science. 290: $147-150$

35. Wilson, B.D., et al. 1999. Physiological and anatomical circuitry between Agouti-related protein and leptin signaling. Endocrinology. 140:2387-2397.

36. Baskin, D.G., Breininger, J.F., and Schwartz, M.W.
1999. Leptin receptor mRNA identifies a subpopulation of neuropeptide $Y$ neurons activated by fasting in rat hypothalamus. Diabetes. 48:828-833.

37. Williams, L.M., et al. 1999. Leptin receptor and neuropeptide $Y$ gene expression in the sheep brain. J. Neuroendocrinol. 11:165-169.

38. Mercer, J.G., et al. 1996. Coexpression of leptin receptor and preproneuropeptide $y$ mRNA in arcuate nucleus of mouse hypothalamus. J. Neuroendocrinol. 8:733-735.

39. Hakansson, M.L., Hulting, A.L., and Meister, B. 1996. Expression of leptin receptor mRNA in the hypothalamic arcuate nucleus-relationship with NPY neurones. Neuroreport. 7:3087-3092.

40. Hentges, S.T., et al. 2004. GABA release from proopiomelanocortin neurons. J. Neurosci. 24:1578-1583.

41. Munzberg, H., Huo, L., Nillni, E.A., Hollenberg, A.N., and Bjorbaek, C. 2003. Role of signal transducer and activator of transcription 3 in regulation of hypothalamic proopiomelanocortin gene expression by leptin. Endocrinology. 144:2121-2131.

42. Banks, A.S., Davis, S.M., Bates, S.H., and Myers, M.G., Jr. 2000. Activation of downstream signals by the long form of the leptin receptor. J. Biol. Chem. 275:14563-14572.

43. Bahrenberg, G., et al. 2002. Identification of the critical sequence elements in the cytoplasmic domain of leptin receptor isoforms required for Janus kinase/signal transducer and activator of transcription activation by receptor heterodimers. Mol. Endocrinol. 16:859-872.

44. Tong, Q., Gamper, N., Medina, J.L., Shapiro, M.S., and Stockand, J.D. 2004. Direct activation of the epithelial $\mathrm{Na}(+)$ channel by phosphatidylinositol 3,4,5-trisphosphate and phosphatidylinositol 3,4-bisphosphate produced by phosphoinositide 3-OH kinase. J. Biol. Chem. 279:22654-22663.

45. Viard, P., et al. 2004. PI3K promotes voltage-dependent calcium channel trafficking to the plasma membrane. Nat. Neurosci. 7:939-946.

46. Balthasar, N., et al. 2004. Leptin receptor signaling in POMC neurons is required for normal body weight homeostasis. Neuron. 42:983-991.

47. Challis, B.G., et al. 2004. Mice lacking proopiomelanocortin are sensitive to high-fat feeding but respond normally to the acute anorectic effects of peptide-YY(3-36). Proc. Natl. Acad. Sci. U. S. A. 101:4695-4700.

48. Zhang, Y., Muyrers, J.P., Testa, G., and Stewart, A.F. 2000. DNA cloning by homologous recombination in Escherichia coli. Nat. Biotechnol. 18:1314-1317.

49. Mercer, E.H., Hoyle, G.W., Kapur, R.P., Brinster, R.L., and Palmiter, R.D. 1991. The dopamine betahydroxylase gene promoter directs expression of E. coli lacZ to sympathetic and other neurons in adult transgenic mice. Neuron. 7:703-716.

50. Takeda, K., et al. 1997. Targeted disruption of the mouse Stat3 gene leads to early embryonic lethality. Proc. Natl. Acad. Sci. U. S. A. 94:3801-3804.

51. He, T.C., et al. 1998. A simplified system for generating recombinant adenoviruses. Proc. Natl. Acad. Sci. U. S. A. 95:2509-2514. 